\title{
More Than Just a Weed: An Exploration of the Antimicrobial Activity of Rumex crispus using a Multivariate Data Analysis Approach"
}

\author{
Authors \\ Affiliations \\ 1 Department of Chemistry and Biochemistry, The Univer- \\ sity of North Carolina at Greensboro, Greensboro NC, \\ United States \\ 2 Department of Pharmaceutical Sciences, University of Illi- \\ nois at Chicago, Chicago IL, United States
}

Chantal V. Pelzer ${ }^{1}$, Joëlle Houriet ${ }^{1}$, William J. Crandall ${ }^{1}$, Daniel A. Todd ${ }^{1}$, Nadja B. Cech ${ }^{1}$, Derick D. Jones Jr. ${ }^{1,2}$

\begin{abstract}
Key words
Botanicals, biochemometrics, selectivity ratio, ultraperformance liquid chromatography, mass spectrometry, Rumex crispus, Polygonaceae
\end{abstract}

\begin{tabular}{|c|c|}
\hline received & July 12, 2021 \\
\hline accepted after revision & September 15, 2021 \\
\hline published online & October 25, 2021 \\
\hline \multicolumn{2}{|l|}{ Bibliography } \\
\hline \multicolumn{2}{|c|}{ Planta Med 2022; 88: 753-761 } \\
\hline \multicolumn{2}{|c|}{ DOI $\quad 10.1055 / a-1652-1547$} \\
\hline \multicolumn{2}{|l|}{ ISSN $\quad 0032-0943$} \\
\hline \multicolumn{2}{|c|}{ (c) 2021. Thieme. All rights reserved. } \\
\hline \multirow{2}{*}{\multicolumn{2}{|c|}{$\begin{array}{l}\text { Georg Thieme Verlag KG, Rüdigerstraße 14, } \\
70469 \text { Stuttgart, Germany }\end{array}$}} \\
\hline & \\
\hline \multicolumn{2}{|l|}{ Correspondence } \\
\hline \multicolumn{2}{|l|}{ Dr. Derick D. Jones Jr. } \\
\hline \multirow{2}{*}{\multicolumn{2}{|c|}{$\begin{array}{l}\text { Department of Chemistry and } \\
\text { North Carolina at Greensboro }\end{array}$}} \\
\hline & \\
\hline \multirow{2}{*}{\multicolumn{2}{|c|}{$\begin{array}{l}301 \text { Mclver St., Sullivan Scienc } \\
\text { 27402, United States }\end{array}$}} \\
\hline & \\
\hline \multicolumn{2}{|c|}{$\begin{array}{l}\text { Phone: + } 125241281 \text { 82, Fax: + } 13363345402 \\
\text { ddiones4@uic.edu }\end{array}$} \\
\hline
\end{tabular}

$\Theta$ Supplementary material is available under https://doi.org/10.1055/a-1652-1547

\begin{abstract}
Plants have a long history of use for their medicinal properties. The complexity of botanical extracts presents unique challenges and necessitates the application of innovative approaches to correctly identify and quantify bioactive compounds. For this study, we used untargeted metabolomics to explore the antimicrobial activity of Rumex crispus (yellow dock), a member of the Polygonaceae family used as an herbal remedy for bacterial infections. Ultra-performance liquid chromatography coupled with high resolution mass-spectrometry (UPLC-MS) was used to identify and quantify the known antimicrobial compound emodin. In addition, we used biochemometric approaches to integrate data measuring antimicrobial activity from $R$. crispus root starting material and fractions against methicillin-resistant Staphylococcus aureus (MRSA) with UPLC-MS data. Our results support the hypothesis that multiple constituents, including the anthraquinone emodin, contribute to the antimicrobial activity of R. crispus against MRSA.
\end{abstract}

\section{Introduction}

Botanicals are a major source of medicinally relevant compounds. Medicines from plant-based sources (botanicals) make an important contribution to the healthcare industry [1]. However, the complexity of botanical extracts makes separation, purification, and identification of their active constituents challenging. The purpose of this study was to employ analytical strategies that address this complexity to evaluate the antimicrobial activity of the botanical medicine yellow dock (Rumex crispus). $R$. crispus is a member of the Polygonaceae family, originally native to Europe and Asia, but now also found in the Americas [2]. It is a common weed that grows in grassy areas, on roadsides, and near sand dunes [2]. The Rumex genus contains roughly 200 species [3]. $R$. crispus has extensive history of being used as an herbal remedy, and is thought to have beneficial properties for people suffering from chronic skin problems in many traditional medical practices [3]. The root of this plant has been used as a poultice or dried powder to treat sores, ulcers, wounds, and various other skin dis-

\# Dedicated to Professor Dr. A. Douglas Kinghorn on the occasion of his 75th birthday. 

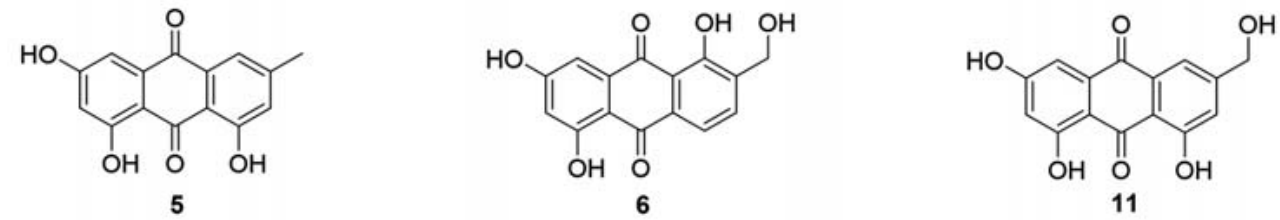

- Fig. 1 Structures of polyhydroxyanthraquinones detected in the $R$. crispus extract and fractions. Compound 5 is emodin (1,3,8-trihydroxy-6methylanthraquinone), compound 6 is 1,3,5-trihydroxy-6-hydroxymethylanthraquinone, and compound 11 is omega-hydroxyemodin (1,3,8-trihydroxy-6-hydroxymethlyanthraquinone). The structures of 5 and 11 were confirmed by matching accurate mass, retention time, and fragmentation patterns with standards (Supplemental Fig. $2 \mathrm{~S}$ and $4 \mathrm{~S}$ ). The structural assignment of 6 is putative only, based on matching accurate mass of the compound with the same molecular formula that has previously been reported to be a constituent of $R$. crispus. The features corresponding to 5 and 6 were associated with the antimicrobial activity of $R$. crispus.

orders [3]. This traditional use is supported by reported in vitro antimicrobial activity of $R$. crispus extracts $[4,5]$.

Anthraquinones are a chemical class of compounds found in $R$. crispus [6]. Of the anthraquinones from $R$. crispus, emodin (1,3,8-trihydroxy-6-methylanthraquinone) ( $\triangleright$ Fig. 1) [7] is the most widely studied, and has also found in multiple other plant species [8]. The antimicrobial properties of $R$. crispus and its constituents are relevant given that it has shown activity against methicillin-resistant Staphylococcus aureus (MRSA) [9], a drug-resistant bacterium that has been identified as a pathogen of international concern by the World Health Organization [10]. In particular, community-associated infections of MRSA have recently emerged as a rising concern [11]. Given that traditional $R$. crispus preparations used to treat skin infections are complex mixtures, we hypothesized that the antimicrobial activity of this botanical could be due to the combined activity of multiple compounds. To test this hypothesis, we employed bioassay guided fractionation in combination with biochemometrics to identify compounds associated with the antimicrobial activity of $R$. crispus against MRSA.

\section{Results and Discussion}

Chloramphenicol [12] and emodin [13] served as controls in this study, and their minimum inhibitory concentrations (MICs) were evaluated and used for comparison between this study and published literature [12]. The tested concentration chloramphenicol ranged from 0 to $200 \mu \mathrm{g} / \mathrm{mL}$ ( 0 to $619 \mu \mathrm{M}$ ) and it displayed an MIC of $12.5 \mu \mathrm{g} / \mathrm{mL}(38.7 \mu \mathrm{M})$ (Supplementary Fig. 1SA). Due to limited solubility, $200 \mu \mathrm{g} / \mathrm{mL}(740 \mu \mathrm{M})$ was the highest emodin concentration tested, but growth inhibition was $>95 \%$ at this concentration, thus $200 \mu \mathrm{g} / \mathrm{mL}(740 \mu \mathrm{M})$ was defined as the MIC of this compound (Supplementary Fig. 1SB).

Antimicrobial activity of a series of fractions (1-10) from a complex R. crispus extract starting material (SM) at two concentrations (10 and $100 \mu \mathrm{g} / \mathrm{mL}$ ) was assessed against MRSA ( $\bullet$ Fig. 2a). Percent inhibition of MRSA using R. crispus SM and fractions was calculated and samples with values $>50 \%$ at $100 \mathrm{\mu g} / \mathrm{mL}$ were defined as active, which include fractions 3-7 ( $\vee$ Fig. 2 a). Fraction 3 demonstrated the most potent antimicrobial activity, with a percent inhibition of $92 \%$ at $100 \mu \mathrm{g} / \mathrm{mL}$ Fractions 4-7 also inhibited MRSA growth ( $\bullet$ Fig. $\mathbf{2 a}$ ) in a dose depen- dent fashion, but their activity was not as potent as that of fraction 3.

The purpose of this study was to test the hypothesis that multiple compounds contribute to the antimicrobial activity of $R$. crispus. Given that emodin is a known constituent of $R$. crispus with antimicrobial activity, our first step was to determine how much

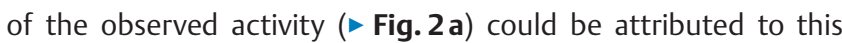
compound. UPLC-MS/MS was used to identify and quantify emodin in the fractions (Supplementary Fig. 2S, Table 1S). The predicted antimicrobial activity ( $\bullet$ Fig. $\mathbf{2}$ b) was then calculated for each fraction using Equation 1. This method of predicting activity based on the concentration of a known antimicrobial compound is similar to that reported by Caesar et. al. [14]. Notably, in the referenced study [14], the known antimicrobial constituent was intentionally spiked into each of the fractions. Spiking was not necessary in the current study because emodin was detected in multiple fractions (fractions 3-10 and starting material, Supplementary Table 1S).

The data comparing predicted and observed activity ( $\bullet$ Fig. $\mathbf{2 b}$ ) can be interpreted as follows. Fractions with lower observed $\%$ inhibition values than predicted values indicate potential synergistic or additive effects between emodin and other constituents of $R$. crispus. Although fraction 3 demonstrated similar observed and predicted activity and had the highest quantity of emodin, the predicted activity of fractions 4, 5, 6 and 7 was lower than the observed activity for these fractions. Thus, it is possible that other constituents in addition to emodin contribute to the antimicrobial activity of these fractions.

Biochemometric analysis was employed to integrate biological and chemical datasets [15]. Supervised analysis of metabolomics data was carried out using orthogonal partial least squares discriminant analysis (OPLS-DA). The purpose of this analysis was to determine which features (defined by their mass to charge ratio $(\mathrm{m} / \mathrm{z})$ and retention time (RT)) detected in the $R$. crispus extract were most strongly associated with antimicrobial activity against MRSA, and to propose possible constituents that might be responsible for the greater-than-predicted antimicrobial activity of fractions 4-7. Bacterial growth inhibition as measured by the \% inhibition was used to guide the analysis, serving as the dependent variable. It is notable that this approach of correlating activity with the distribution of compounds across fractions works best when compounds are present in multiple fractions. Such a scenario is 
Rumex crispus antimicrobial assessment

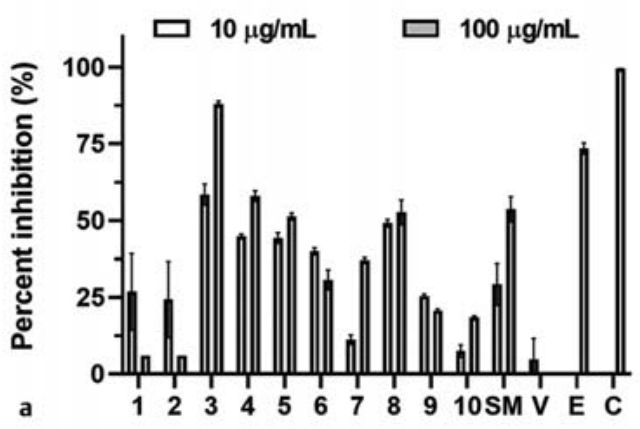

Predicted and observed antimicrobial activity

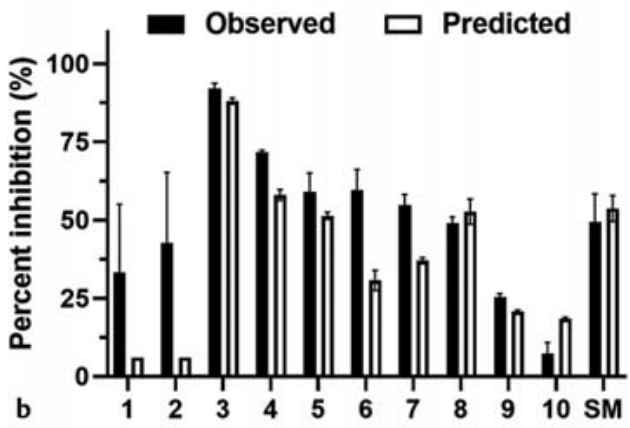

- Fig. 2 a Antimicrobial activity of $R$. crispus roots fractions $1-10$ and extract/starting material (SM) at concentrations $100 \mu \mathrm{g} / \mathrm{mL}$ and $10 \mu \mathrm{g} / \mathrm{mL}$ (expressed as mass of extract or fraction per assay well volume) with DMSO as vehicle. Data are also included for vehicle (V, $2 \%$ DMSO) and purified emodin $(E, 100 \mu \mathrm{g} / \mathrm{mL}$ ) and chloramphenicol (C, $100 \mu \mathrm{g} / \mathrm{mL})$. Full dose-response curves for emodin and chloramphenicol are available as Supplemental Fig. 1S. Fractions 3-7 are the most active against MRSA LAC USA300 with percent inhibition > 50\%. b Comparison of predicted and observed antimicrobial activity of $R$. crispus fractions $1-10$ and starting material (SM) at an assay concentration of $100 \mu \mathrm{g} / \mathrm{mL}$ based on the content of emodin in each. Activity was evaluated against MRSA LAC USA300. Fraction 3 has similar observed and predicted values, however active fractions 4-7 have greater observed than predicted activity.
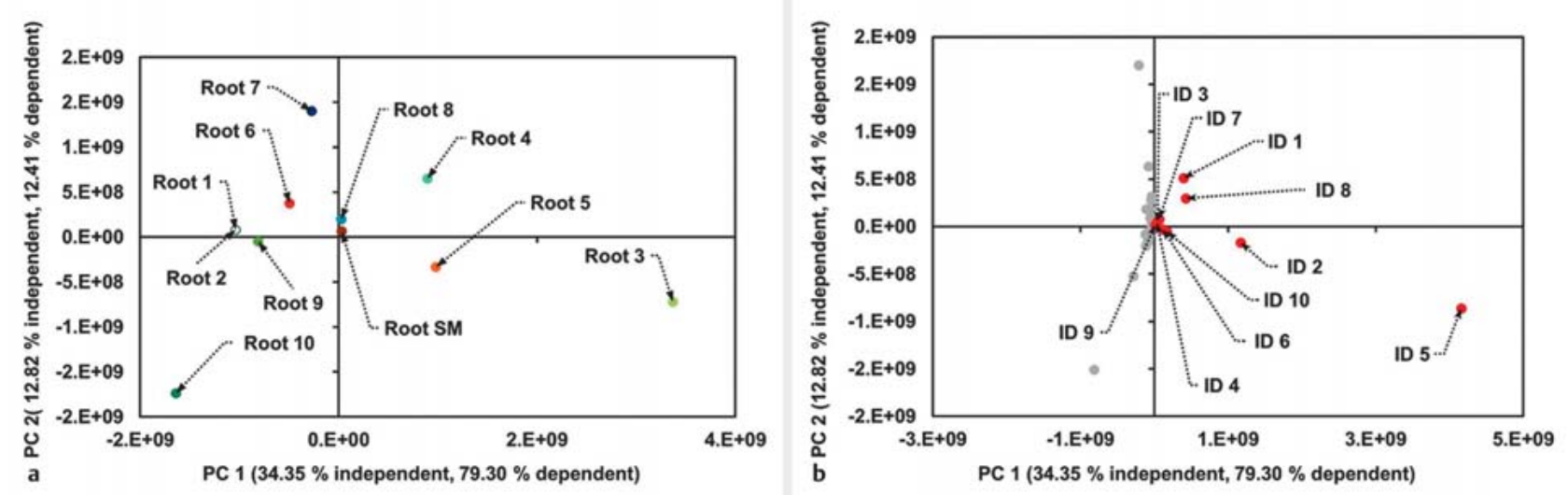

- Fig. 3 Orthogonal partial least squares discriminant analysis (OPLS-DA) scores plot (a) and loadings plot (b) of $R$. crispus fractions 1-10 and SM tested for antimicrobial activity against Staphylococcus aureus at $100 \mathrm{\mu g} / \mathrm{mL}$. MRSA growth inhibition was measured in triplicate using percent inhibition. The fractions, extract and pure compounds were analyzed with UPLC-HRMS (via an Acquity UPLC and Q-Exactive orbitrap mass spectrometer) in triplicate. An independent chemical analysis by UPLC-MS was conducted for each solution measured in the antimicrobial assay. Datapoints shown in green also demonstrated selectivity ratio values (SR) greater than 0.1 and are highlighted in > Fig. 4.

common when the separation is accomplished at the preparative scale with flash chromatography, as was the case with this study.

The loadings plot ( $\vee$ Fig. $\mathbf{3}$ b) depicts the features with their position in the plot demonstrating to what extent they explain the difference among samples. Fraction 3, which demonstrated the most pronounced antimicrobial activity of those tested, is shown in the far right of the scores plot ( $\vee$ Fig. 3 a), separated from the other fractions. The features represented in the right-most region of the loadings plot ( $\vee$ Fig. $\mathbf{3} \mathbf{b}$ ) are therefore, expected to be most strongly associated with antimicrobial activity.

Another way of displaying the correlation between biological activity and chemical composition is through a selectivity ratio plot ( $\bullet$ Fig. 4). The selectivity ratio can be used as a ranking tool to determine which features of a given mixture are associated most strongly associated with biological activity [15-18]. The selectivity ratio is defined as the quotient of the explained variance and the unexplained variance in the dataset [19]. Features that are correlated with measured activity will have higher selectivity ratios. A total of ten features were identified as having selectivity ratios above the empirically determine cut-off value of 0.10 . Implementation of the selectivity ratio plot is advantageous because it minimizes the impact of the heteroscedastic noise and facilitates the detection of relevant features that give only weak signals in the mass spectral data $[15,16]$. Thus, the use of the selectivity ra- 


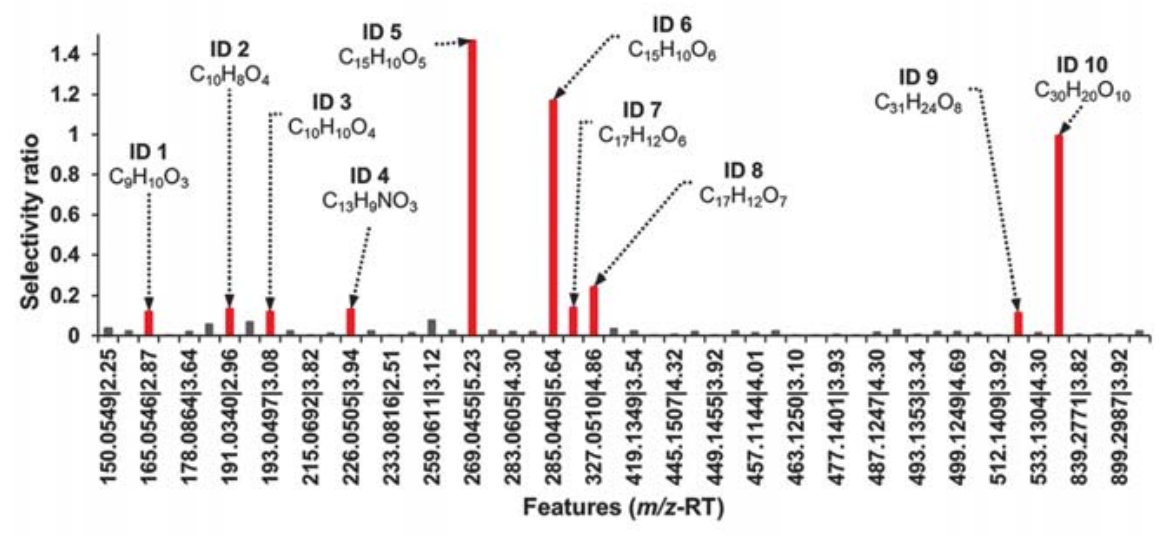

- Fig. 4 Selectivity ratio plot of R. crispus roots fractions $1-10$ and starting material (SM) at $100 \mu \mathrm{g} / \mathrm{mL}$. This plot shows the multivariate statistical analysis of the untargeted metabolomics dataset and identifies features that correlate with MRSA growth inhibition. Percent growth inhibition of bacterial growth of each fraction or extract were used as the dependent variable. The $x$-axis represents the feature (identified by $m / z$ and retention time) detected. Features are shown in order of increasing mass. Peaks are numbered to correspond with putative annotations $(\triangleright$ Table 1$)$. Higher selectivity ratio indicates stronger correlation with growth inhibition.

tio plot ( $\vee$ Fig. 4 ) enabled identification of features from the $R$. crispus extract that correlated to the inhibition of MRSA growth that were not readily apparent in the loadings plot ( $\bullet$ Fig. $\mathbf{3} \mathbf{b}$ ).

The features with selectivity ratios above 0.10 were annotated ( $\triangleright$ Table 1) based on comparison with standards and assignment of molecular formulae found within the literature. The annotations considered the previous structures described for the genus, species, and family of $R$. crispus. The confidence of annotation was designated by the scoring system described in [20]. Consistent with the data shown in $\mathbf{r}$ Fig. $\mathbf{3}$, feature 5 displayed the most positive selectivity ratio value and corresponded to the $[\mathrm{M}-\mathrm{H}]^{-}$ion of emodin (m/z 269.0457, RT 5.23 min) ( Fig. 4). Excellent agreement was observed between the standard and the putative emodin identified in $R$. crispus (Supplementary Fig. 2S). Thus, emodin and its associated ions were assigned MSI level of confidence of 1 $(\triangleright$ Table 1 ). After feature 5 , the highest selectivity ratio values ( $\vee$ Fig. 4) were observed for features 6 and 10, both of which were present at the highest abundance in Fraction $3(\triangleright$ Table 1$)$. Feature 10 was assigned to the proton bound dimer of emodin ([2 M $-\mathrm{H}^{-}, \mathrm{m} / \mathrm{z} 539.0984$ at $5.22 \mathrm{~min}$ ). Identification of the features related to emodin (5 and 10) was confirmed by comparing their $\mathrm{m} / \mathrm{z}$ in negative polarity, retention time, MS/MS fragmentation pattern, and isotope distribution.

Initially, we proposed based on molecular formula $\left(\mathrm{C}_{15} \mathrm{H}_{10} \mathrm{O}_{6}\right)$ that feature 6 ( $m / z-R T 285.0405,5.64)$ could be omega-hydroxyemodin (1,3,8-trihydroxy-6-hydroxymethylanthraquinone), which is a previously reported to have antimicrobial activity [21]. However, although omega-hydroxy-emodin was identified in the R. crispus extract and in fraction 6 (Supporting Fig. 4S), the observed retention time for feature 6 (5.64 $\mathrm{min})$ in fraction 3 did not match with that of an omega-hydroxy-emodin standard (3.66 min). Thus, feature 6 appears to be an isomer of omega-hydroxy-emodin, and is tentatively (MSI level 2 ) assigned the identity of 1,3,5-trihydroxy-6-hydroxymethylanthraquionone, a constituent that has previously been reported in $R$. crispus [6] ( $\bullet$ Ta- ble 1). Comparison of the structures of emodin (5), 1,3,5-trihydroxy-6-hydroxymethylanthraquionone (6) and omega-hydroxyemodin (11) are shown in > Fig. 1. Further experiments would be required to isolate the compound corresponding to feature 6 and confirm its structure. Omega-hydroxy-emodin (11) appeared not to be associated with the observed antimicrobial activity of the $R$. crispus extract, given that its corresponding feature was not assigned a selectivity ratio value of $>0.1$.

Seven other features identified by the selectivity ratio plot as correlating with biological activity of $R$. crispus were most abundant in fraction 4. Among them, two compounds matching the calculated molecular formulae have been previously described for the genus Rumex (1 [3, 22], 3 [23]) while four were described for the family Polygonaceae (2 [24], 7 [25], 8 [26], 9 [27]). Among these annotated features, several have been shown to have relevant biological effects. Compound 2 (scopoletin) has been reported as antimicrobial and antifungal [28], compound 3 (isoferulic acid) has demonstrated weak antimicrobial activity against E. coli [29], and compound 8 (laccaic acid D methyl ester) has been shown to inhibit Mycobacterium tuberculosis [30]. The reported antimicrobial effects of these compounds support the hypothesis that they may play a role in antimicrobial activity of Rumex crispus. However, most of the reported literature for the putative active compound does not directly report on activity against MRSA, and a study of the activity of scopoletin against one strain of MRSA showed it to be inactive [31]. Further experiments would be necessary to isolate the putatively identified features ( $\triangleright$ Table 1 ), confirm the tentative structural assignments, and evaluate antimicrobial activity of the purified compounds against the MRSA strain investigated here (USA300 LAC). It is possible that these compounds are active only in mixtures; thus, it would be of value to test their activity both alone and in combination with emodin.

Collectively, the results of these studies, consistent with literature [4], confirm previous work suggesting a role of emodin in the 


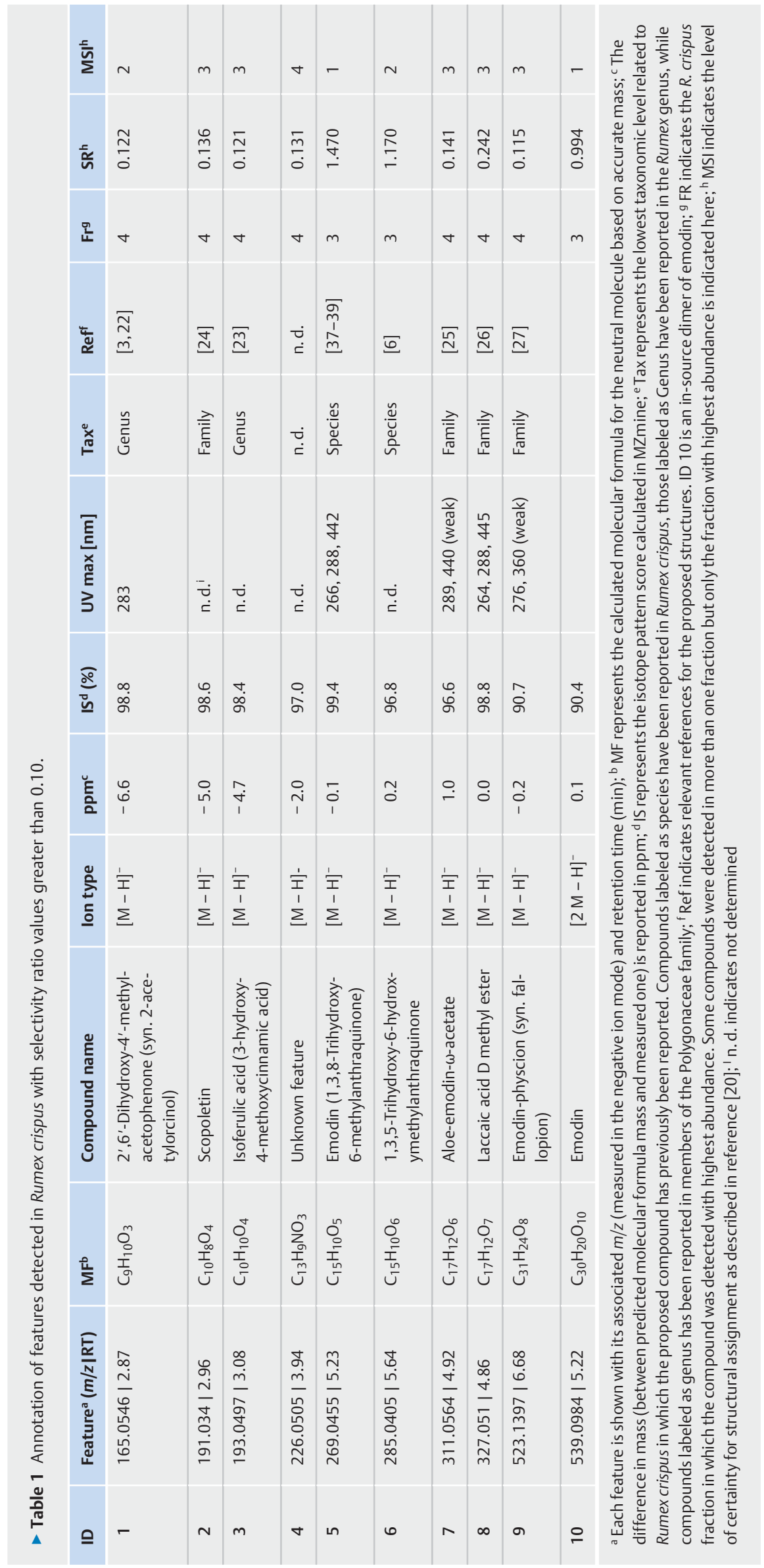


activity of $R$. crispus against MRSA. We also predicted that the antimicrobial activity of this botanical could not be attributed to emodin alone. Informatics analysis of the antimicrobial data support this prediction and suggest that an array of small molecules from $R$. crispus work collectively to exert antimicrobial effects against MRSA. These findings are consistent with the traditional use of Rumex crispus topically as a complex mixture and suggest that such a mixture may be more effective against MRSA than a single compound alone. However, the studies presented herein are in vitro only, and they should not be extrapolated directly to draw conclusions about the efficacy of $R$. crispus for use in humans. Importantly, there may be toxicity concerns with the oral consumption of emodin and related compounds in botanical preparations [7]. In light of this, the European Union currently requires processing procedures to remove emodin for botanical preparations known to contain high levels of this compound prior to human consumption [32]. Whether or not toxicity concerns are relevant to topical use of emodin containing botanicals such as $R$. crispus could be a topic of future investigation.

\section{Materials and Methods}

\section{Plant Material}

Fresh roots of $R$. crispus were collected on June 20, 2019 from the University of North Carolina at Greensboro Garden in Greensboro, North Carolina ( $\left.\mathrm{N} 36.07^{\circ}, \mathrm{W} 79.81^{\circ}\right)$ and identity was confirmed by Nadja B. Cech. A voucher specimen of $R$. crispus roots was deposited at the herbarium of the University of North Carolina at Chapel Hill (NCU671166).

\section{Extraction}

Fresh $R$. crispus roots were dried in a single wall transite oven (Blue $\mathrm{M}$ Electric Company) at $37^{\circ} \mathrm{C}$ for 24 hours. The dry mass of the resulting starting material $(64.00 \mathrm{~g})$ was ground using a Wiley Mill Standard Model No. 3 (Arthur Thomas Company) and submerged in methanol (MeOH) (Fisher Scientific, ACS grade) at $160 \mathrm{~g} / \mathrm{L}$ for 24 hours. The resulting extract was decanted and dried in a round bottom flask (Fisher) using a rotary evaporator (G3 Heidolph, Heidolph Instruments). Plant material was resubmerged for 24 hours with the $\mathrm{MeOH}$ recycled from the rotary evaporator. The extraction process was repeated once more. During the last extraction, the $\mathrm{MeOH}$ was decanted as before without drying down. Doubly deionized water $\left(\mathrm{H}_{2} \mathrm{O}\right)$ was added to $\mathrm{MeOH}\left(9: 1 \mathrm{MeOH}: \mathrm{H}_{2} \mathrm{O}\right)$ and then sonicated to dissolve the three pooled extractions of the plant material in the same round bottom flask. The resulting mixture was partitioned with hexane (1:1 MeOH: $\mathrm{H}_{2} \mathrm{O}$ : hexane). The obtained aqueous $\mathrm{MeOH}$ layer was partitioned further with ACS grade ethyl acetate (EtOAc) (Fisher Scientific) empirically (4:1 EtOAc: $\mathrm{MeOH} / \mathrm{H}_{2} \mathrm{O}$ ). To remove hydrosoluble tannins, the EtOAc layer was washed with a $1 \%$ sodium chloride $(\mathrm{NaCl})$ solution. The $\mathrm{NaCl}$ solution was made using $\mathrm{dd}_{2} \mathrm{O}$ and $\mathrm{NaCl}$ pellets (Fisher Scientific). The resulting EtOAc layer was dried under nitrogen, yielding $2.47 \mathrm{~g}$ dried extract (3.86\% yield), before chromatographic separation. The extraction scheme can be visualized in Fig. 5S (Supporting Information).

\section{Flash Chromatography}

Flash chromatographic separations were completed using a CombiFlash RF system (Teledyne-Isco) coupled to a PDA detector and an evaporative light scattering detector (ELSD). The first-stage separation of the EtOAc extract (2.47 g) was conducted using normal-stage flash chromatography (Fig.5S, Supporting Information) on a $40 \mathrm{~g}$ silica gel column. The ELSD was set to 55 and pressure of 1.5 bar. The nitrogen gauge was set at 20 psi. A $40 \mathrm{~mL} / \mathrm{min}$ flow rate was used and a total column volume (CV) of 89.2 over a hexane $/ \mathrm{CHCl}_{3} / \mathrm{MeOH}$ gradient. The gradient consisted of ACS grade chloroform $\left(\mathrm{CHCl}_{3}\right)$ (Fisher Scientific) as solvent $\mathrm{A} 1, \mathrm{MeOH}$ as solvent $\mathrm{B} 1$, and hexane as solvent $\mathrm{B} 2$. The flash chromatographic system runs a dual solvent system and changes solvent within the duration of the run. At the beginning of the run, an isocratic step with $100 \%$ of B2, was held for 5 CVs. The gradient (A1: B2) began at 5 CVs as B2 decreased to $0 \%$ for 22 CVs. B2 was switched to $B 1$ for 5 CVs. The gradient (A1:B1) increased to $0: 100$ for 32 CVs and B1 was held isocratically at $100 \%$ for the last 25.2 CVs.

\section{UPLC-MS Analysis}

UPLC-MS analysis was conducted in negative mode using a Q-Exactive Plus mass spectrometer (Thermo Fisher Scientific) connected to an Acquity UPLC system (Waters Corporation). When collecting UPLC-MS data, $3 \mu \mathrm{L}$ of $1 \mathrm{mg} / \mathrm{mL}$ samples suspended in $\mathrm{MeOH}$ were injected into the column. Using a flow rate of $0.3 \mathrm{~mL} / \mathrm{min}$, samples eluted from the column (BEH C18 $1.7 \mu \mathrm{m}$, $2.150 \mathrm{~mm}$, Waters Corporation) using the following gradient with solvent $A$ consisting of water with $0.1 \%$ formic acid and solvent $B$ consisting of acetonitrile with $0.1 \%$ formic acid: $90: 10$ (A:B) from 0-0.5 min, increasing to $0: 100(A: B)$ from $0.5-8.0$ min. The gradient was isocratically held at $100 \% \mathrm{~B}$ for $0.5 \mathrm{~min}$, before returning to starting conditions over $0.5 \mathrm{~min}$ and held from $9.0-10.0 \mathrm{~min}$. Mass analysis was completed in negative ionization mode over a scan range of 150-1500 with the following settings: spray voltage at $3.20 \mathrm{kV}$, capillary temperature at $256^{\circ} \mathrm{C}$, sheath gas flow at 47 , auxiliary gas flow at 11 , spare gas flow at 2 , and an S-lens RF level of 50. MS/MS data were collected using the same procedure with the exception of using the LTQ XL Orbitrap Mass Spectrometer with the following settings: spray voltage at $4.00 \mathrm{kV}$, capillary temperature at $325^{\circ} \mathrm{C}$, capillary voltage at $20 \mathrm{~V}$, sheath gas flow at 30 , auxiliary gas flow at 15 , sweep gas flow at 1 , and tubes lens at $100 \mathrm{~V}$.

\section{Quantification of emodin and prediction of antimicrobial activity}

A calibration curve of emodin was constructed to quantify the amount of emodin in the extract and its subfractions. Emodin standards were prepared at a range of $1.00 \times 10^{-3}$ to $1.00 \times$ $10^{2} \mathrm{\mu g} / \mathrm{mL}$ in optima grade methanol (Fisher-Scientific and analyzed by UPLC-HRMS as described below. The regression coefficient $\left(R^{2}\right)$ value of the calibration curve of emodin is reported as 0.9961. Emodin concentration was calculated from the best fit line obtained by linear regression analysis of this calibration curve (Fig. 3S, Supporting Information). The limit of detection for the analysis (LOD) was defined as the lowest concentration in the cal- 
ibration curve for which the residual (difference between experimental and calculated concentration) was less than $25 \%$.

The predicted antimicrobial activity of emodin was calculated from Equation 1 based on its concentration in each extract and fraction and the dose-response relationship for growth inhibition of Staphylococcus aureus ( $\triangleright$ Fig. $\mathbf{2}$ b). In Equation 1, y is the predicted biological response emodin will have at a given concentration, $\mathrm{min}$ is the bottom of the dose-response curve $(-0.2205)$, max is the top of the curve (0.9280), the hillslope is a numerical value that characterizes the steepness of the slope at the midpoint $(-0.603)$, and $x$ is the concentration of emodin in each extract and fraction (Supplementary Table 1S). This approach is similar to that employed by Caesar et al. to predict antimicrobial activity of botanical extracts [14].

$y=\min +\frac{\max -\min }{1+\left(\frac{\mathrm{x}}{\mathrm{IC}_{50}}\right)^{- \text {hillslope }}}$

\section{Untargeted Metabolomics Analysis}

UPLC-HRMS data were collected in negative mode and the raw data from these analyses are publicly available via MassIVE (https://doi.org/doi:10.25345/C5K829). The data were analyzed, aligned, and filtered utilizing MZmine 2.53 (http://mzmine. sourceforge.net/) [16,33]. ThermoRAW MS data from first-stage fractions were converted to. mzXML using ProteoWizard [34] and uploaded for peak picking into MZmine. During mass detection, the ions retained were those above a noise level of $4 \times 10^{4}$. Chromatogram builder was employed with a minimum group size of 5 scans, a group intensity threshold of $4 \times 10^{4}$, a minimum height of $1 \times 10^{6}$ and $\mathrm{m} / \mathrm{z}$ tolerance of $0.003 \mathrm{Da}$. ADAP algorithm was used for deconvolution with the following parameters: signal to noise threshold at 10 , min feature height at $1 \times 10^{6}$, coefficient to area threshold at 100 , peak duration from 0 to $1 \mathrm{~min}$, retention time wavelet range from 0 to $0.10 \mathrm{~min}$. Isotopes were grouped with a $\mathrm{m} / \mathrm{z}$ tolerance of $0.0015 \mathrm{Da}$ and a RT tolerance of $0.05 \mathrm{~min}$. Triplicate analyses were aligned using the join aligner tool with the same $\mathrm{m} / \mathrm{z}$ and RT than for isotope grouping, the weight for $m / z$ was set at 2 and for RT at 1 . Isotope patterns were compared with the same $\mathrm{m} / \mathrm{z}$ tolerance, a minimum height of $4 \times 10^{4}$ and a minimum score of $50 \%$. Triplicate analyses were filtered to keep peaks detected in all triplicate. Blank analyses $(n=15)$ were aligned with the same parameters and filtered to keep the peaks detected in at least 7 blanks. All triplicates' series and blanks were subsequently aligned, and peaks detected in the blanks were deleted. The final peak lists contained 541 peaks. The resulting data matrix, consisting of $m / z$, retention time, and peak area, was exported as a .csv file to Excel (Microsoft). Filtered chemical data was merged with MRSA inhibition data from samples tested at $100 \mu \mathrm{g} / \mathrm{mL}$ to form the final data set for OPLS-DA and selectivity ratio analysis.

Multivariate analyses were performed with Sirius 11.5 statistical software (Pattern Recognition Systems) [16]. An internally cross-validated PLS model was produced using 100 iterations and a significance level of 0.05 . Statistical algorithms internal to the Sirius software utilized model predictions to produce selectivity ratios identifying putative antimicrobial constituents. Peaks with an SR value of 0 (no correlation detected) were filtered out to give a total of 52 highlighted features of which those with a minimum SR value of 0.10 were annotated ( 10 features) in $>$ Table 1 . These features were annotated based on UPLC-MS data and previously published data for $R$. crispus. The annotations were presented according to the level of confidence score proposed in the metabolomics standard initiative (MSI) [20] and ranged between 1 and 4 (with 1 being higher confidence of identification).

\section{Antimicrobial Assay}

To evaluate antimicrobial activity, broth dilution assays were conducted by assessing growth inhibition of a clinically relevant strain of methicillin-resistant S. aureus (MRSA USA300 LAC strain AH1263) using Clinical Laboratory Standards Institutes (CLSI) methods [35]. This strain was obtained from Dr. Alexander Horswill at the University of Colorado Anschutz Medical Campus. Cultures were grown from a single colony isolate of MRSA in Müeller-Hinton broth (MHB) and diluted to $1.0 \times 10^{5} \mathrm{CFU} / \mathrm{mL}$ based on absorbance at $600 \mathrm{~nm}\left(\mathrm{OD}_{600}\right)$.

$R$. crispus roots extract and fractions were tested in triplicate at final concentrations of 10 and $100 \mu \mathrm{g} / \mathrm{mL}$. Samples were dissolved in microbiological grade dimethyl sulfoxide (DMSO) (Fisher Scientific) and diluted with Mueller Hinton Broth (MHB, Sigma-Aldrich) to prepare final concentrations in broth with less than $2 \%$ DMSO. The known antibiotic chloramphenicol (98\% purity, Sigma-Aldrich) was used as a positive control at the same concentrations as tested extracts and fractions. The vehicle control was $2 \%$ DMSO in MHB. Blank wells consisted of samples at concentrations of 10 and $100 \mu \mathrm{g} / \mathrm{mL}$ without bacteria to enable subtraction of absorbance due to the samples. Wells were inoculated with MRSA, USA300 LAC strain AH1263 [36] and incubated for 24 hours at $37^{\circ} \mathrm{C} . \mathrm{OD}_{600}$ was evaluated after incubation.

MICs were calculated for emodin (analytical standard, $\geq 97 \%$ purity, Sigma-Aldrich) and chloramphenicol (positive control, $98 \%$ purity, Sigma-Aldrich). After a 24 -hour incubation at $37^{\circ} \mathrm{C}$, $\mathrm{OD}_{600}$ values were measured of the emodin and chloramphenicol with a concentration range of $0-200 \mu \mathrm{g} / \mathrm{mL}$. For the purpose of this study, the MIC is defined here as the concentration at which bacterial growth is $<95 \%$ that of the vehicle control. The inhibitory concentration at which bacterial growth is inhibited by $50 \%$, known as the $\mathrm{IC}_{50}$ or $\mathrm{EC}_{50}$, was also calculated to properly predict activity of emodin based on the concentration of emodin in each sample. These values were determined using GraphPad prism software (Version 8.4.3).

\section{Supporting Information}

The dose response curves, calibration curve, MS/MS data and an extraction flowchart are available in the Supporting Information.

\section{Contributors' Statement}

Data collection: D.D. Jones, C.V. Pelzer, D. A. Todd; design of study: D. D. Jones, N. B. Cech, C. V. Pelzer, J. Houriet, D. A. Todd; statistical analysis: D.D. Jones, C.V. Pelzer, J. Houriet; analysis and interpretation of data: W. J. Crandall, D.D. Jones, C. V. Pelzer, D. A. Todd, N.B. Cech; drafting of the manuscript: D.D. Jones, C. V. Pelzer, W. J. Crandall, J. Houriet, D. A. Todd, J. Houriet; critical revision of the manuscript: D.D. Jones, C.V. Pelzer, D. A. Todd, N. B. Cech, J. Houriet. 


\section{Acknowledgements}

This research was supported by the National Center for Complementary and Integrative Health of the National Institutes of Health under award numbers R15 AT010191 (NBC, CVP), and 5 T32 AT008938 (NBC, DDJJ), and by the National Institute for General Medical Sciences of the National Institutes of Health under award number K12GM139186 to DDJJ. The authors thank Dr. Alexander Horswill for providing MRSA strains utilized for this project. Mass spectrometry analyses were conducted at the Triad Mass Spectrometry Facility.

Conflict of Interest

The authors declare that there are no conflicts of interest.

\section{References}

[1] Chugh NA, Bali S, Koul A. Integration of botanicals in contemporary medicine: Road blocks, checkpoints and go-ahead signals. Integr Med Res 2018; 7: 109-125

[2] Zaller JG. Ecology and non-chemical control of Rumex crispus and R. obtusifolius (Polygonaceae): A review. Weed Res 2004; 44: 414-432

[3] Vasas A, Orbán-Gyapai O, Hohmann J. The genus Rumex: Review of traditional uses, phytochemistry and pharmacology. J Ethnopharm 2015; 175: $198-228$

[4] Wegiera M, Kosikowska U, Malm A, Smolarz HD. Antimicrobial activity of the extracts from fruits of Rumex L. species. Cent Eur J Biol 2011; 6: 1036-1043

[5] Yildirim A, Mavi A, Kara AA. Determination of antioxidant and antimicrobial activities of Rumex crispus L. extracts. J Agric Food Chem 2001; 49: 4083-4089

[6] Günaydin K, Topçu G, Ion RM. 1, 5-Dihydroxyanthraquinones and an anthrone from roots of Rumex crispus. Nat Prod Lett 2002; 16: 65-70

[7] Dong X, Fu J, Yin X, Cao S, Li X, Lin L, Huyiligeqi, Ni J. Emodin: A review of its pharmacology, toxicity and pharmacokinetics. Phytother Res 2016; 30: $1207-1218$

[8] Izhaki I. Emodin - a secondary metabolite with multiple ecological functions in higher plants. New Phytol 2002; 155: 205-217

[9] Orbán-Gyapai O, Liktor-Busa E, Kúsz N, Stefkó D, Urbán E, Hohmann J, Vasas A. Antibacterial screening of Rumex species native to the Carpathian Basin and bioactivity-guided isolation of compounds from Rumex aquaticus. Fitoterapia 2017; 118: 101-106

[10] World Health Organization. Antimicrobial Resistance: Global Report on Surveillance. Geneva: World Health Organization; 2014

[11] Otto M. Community-associated MRSA: What makes them special? Int J Med Microbiol 2013; 303: 324-330

[12] Rebstock MC, Crooks HM, Controulis J, Bartz QR. Chloramphenicol (Chloromycetin).1IV.1a Chemical Studies. J Am Chem Soc 1949; 71: 2458-2462

[13] Zhang W, Chen H, Liu DL, Li H, Luo J, Zhang JH, Li Y, Chen KJ, Tong HF, Lin SZ. Emodin sensitizes the gemcitabine-resistant cell line Bxpc-3/Gem to gemcitabine via downregulation of NF-KB and its regulated targets. Int J Oncol 2013; 42: 1189-1196

[14] Caesar LK, Nogo S, Naphen CN, Cech NB. Simplify: A mass spectrometry metabolomics approach to identify additives and synergists from complex mixtures. Anal Chem 2019; 91: 11297-11305

[15] Kellogg J], Todd DA, Egan JM, Raja HA, Oberlies NH, Kvalheim OM, Cech NB. Biochemometrics for natural products research: Comparison of data analysis approaches and application to identification of bioactive compounds. J Nat Prod 2016; 79: 376-386
[16] Kvalheim OM, Brakstad F, Liang Y. Preprocessing of analytical profiles in the presence of homoscedastic or heteroscedastic noise. Anal Chem 1994; 66: 43-51

[17] Kvalheim OM, Karstang TV. Interpretation of latent-variable regression models. Chemometr Intell Lab Syst 1989; 7: 39-51

[18] Jones DD, Caesar LK, Pelzer CV, Crandall W], Jenul C, Todd DA, Horswill AR, Cech NB. Targeted and untargeted analysis of secondary metabolites to monitor growth and quorum sensing inhibition for methicillin-resistant Staphylococcus aureus (MRSA). J Microbiol Met 2020; 176: 106000

[19] Kvalheim OM. Interpretation of partial least squares regression models by means of target projection and selectivity ratio plots. J Chemom 2010; 24: 496-504

[20] Sumner LW, Amberg A, Barrett D, Beale MH, Beger R, Daykin CA, Fan TW, Fiehn O, Goodacre R, Griffin JL, Hankemeier T, Hardy N, Harnly J, Higashi R, Kopka J, Lane AN, Lindon JC, Marriott P, Nicholls AW, Reily MD, Thaden J], Viant MR. Proposed minimum reporting standards for chemical analysis chemical analysis working group (CAWG) metabolomics standards initiative (MSI). Metabolomics 2007; 3: 211-221

[21] Daly SM, Elmore BO, Kavanaugh JS, Triplett KD, Figueroa M, Raja HA, ElElimat T, Crosby HA, Femling JK, Cech NB, Horswill AR, Oberlies NH, Hall PR. $\omega$-Hydroxyemodin limits Staphylococcus aureus quorum sensing-mediated pathogenesis and inflammation. Antimicrob Agents Chemother 2015; 59: 2223-2235

[22] van den Berg AJJ, Labadie RP. The production of acetate derived hydroxyanthraquinones, -dianthrones, -naphthalenes and -benzenes in tissue cultures from Rumex alpinus. Planta Med 1981; 41: 169-173

[23] Hariprasad P, Ramakrishnan N. GC-MS analysis of Rumex vesicarius L. Int J Drug Dev Res 2011; 3: 272-279

[24] Chen X, Wei L, Pu X, Wang Y, Xu Y. Pharmacokinetics and tissue distribution study of 15 ingredients of Polygonum chinense Linn extract in rats by UHPLC-MS/MS. Biomed Chromatogr 2020; e4975

[25] Yang D, Sun G, Zhang A, Fu S, Liu J. Screening and analyzing the potential bioactive components from rhubarb, using a multivariate data processing approach and ultra-high performance liquid chromatography coupled with time-of-flight mass spectrometry. Anal Methods 2015; 7: 650-661

[26] Oshio H, Naruse Y, Tsukui M. Quantitative analysis of the purgative components of rhubarb and senna. Chem Pharm Bull 1978; 26: 2458-2464

[27] Piola F, Bellvert F, Meiffren G, Rouifed S, Walker V, Comte G, Bertrand C. Invasive Fallopia $\times$ bohemica interspecific hybrids display different patterns in secondary metabolites. Écoscience 2013; 20: 230-239

[28] Gnonlonfin GJB, Sanni A, Brimer L. Review Scopoletin - A coumarin phytoalexin with medicinal properties. Crit Rev Plant Sci 2012; 31: 47-56

[29] Kabir F, Katayama S, Tanji N, Nakamura S. Antimicrobial effects of chlorogenic acid and related compounds. J Korean Soc Appl Biol Chem 2014; 57: 359-365

[30] Induli M, Cheloti M, Wasuna A, Wekesa I, Wanjohi JM, Byamukama R, Heydenrich M, Makayoto M, Yenesew A. Naphthoquinones from the roots of Aloe secundiflora. Phytochem Lett 2012; 5: 506-509

[31] Smyth T, Ramachandran VN, Smyth WF. A study of the antimicrobial activity of selected naturally occurring and synthetic coumarins. Int J Antimicrob Agents 2009; 33: 421-426

[32] Commision Regulation (EU) 2018/468. Official journal of the European Union 2021 (March 18, 2021). Accessed October 5, 2021 at: https:// eur-lex.europa.eu/legal-content/EN/TXT/PDF/?uri=CELEX:32021R0468 \&qid $=1616147010900 \&$ from $=$ IT

[33] Pluskal T, Castillo S, Villar-Briones A, Oresic M. MZmine 2: modular framework for processing, visualizing, and analyzing mass spectrometry-based molecular profile data. BMC Bioinformatics 2010; 11: 395

[34] Kessner D, Chambers M, Burke R, Agus D, Mallick P. ProteoWizard: open source software for rapid proteomics tools development. Bioinformatics 2008; 24: 2534-2536 
[35] Humphries RM, Ambler J, Mitchell SL, Castanheira M, Dingle T, Hindler JA, Koeth L, Sei K. CLSI methods development and standardization working group best practices for evaluation of antimicrobial susceptibility tests. J Clin Microbiol 2018; 56: e01934-17

[36] Boles BR, Thoendel M, Roth AJ, Horswill AR. Identification of genes involved in polysaccharide-independent Staphylococcus aureus biofilm formation. Plos One 2010; 5: e10146
[37] Demirezer LO, Uzun M. Determination of sun protection factor (SPF) of Rumex crispus and main anthraquinones. Planta Med 2016; 82: P334

[38] Demirezer ÖL, Kuruüzüm A. Rapid and simple biological activity screening of some Rumex species; Evaluation of bioguided gractions of $R$. scutatus and pure compounds. Z Naturforschung C 1997; 52: 665-669

[39] Başkan S, Daut-Özdemir A, Günaydın K, Erim FB. Analysis of anthraquinones in Rumex crispus by micellar electrokinetic chromatography. Talanta 2007; 71: 747-750 